\title{
An Improved Multi-Stage Method for Medical Image Registration Based on Mutual Information
}

\author{
Maryam Zibaeifard, Mohammad Rahmati \\ Department of Computer Engineering and IT \\ Amirkabir University of Technology, Tehran, Iran \\ zibaeifard@ce.aut.ac.ir, rahmati@aut.ac.ir
}

\begin{abstract}
The objective of registration process is to obtain a spatial transformation of a floating image to a reference image by which a similarity measure is optimized between the two images. A widely used measure is Mutual Information (MI). Registration based on mutual information is robust and dataindependent and could be used for a large class of monomodality and multimodality images. This method requires estimating joint histogram of the two images. As a result, it requires an extremely high computation time. This is its main drawback especially when it is applied to volume images. In order to speed up the registration process, a multi-resolution approach has been introduced before. In this method, a pyramid of low to high resolution images is used to find a rough estimate of parameters of the optimum transformation in a relatively short time using low resolution images, which is subsequently used as initial value for the higher resolutions. An appropriate estimation of the transformation parameters improves speed of the optimization algorithm.

In this paper, we present a new improved method of sample selection for multi-stage registration based on mutual information. Instead of downsampling technique used in the pyramid methods, we propose a new technique to find a suitable subset of image samples, which results in a better estimate of the optimal transformation. A comparison for MR images indicates that our proposed method yields a better registration than subsampling method, especially when subsampling factor is low. Moreover, the experimental results involving three-dimensional clinical images of CT, MR and PET are presented for rigid registration.
\end{abstract}

\section{Introduction}

Image registration is the process of spatial alignment of two or more images acquired from different sensors, viewpoints or time intervals [1]. Registration is widely used in medical imaging applications. A common practice of these applications could be found in fusion of multimodality images when patients have to undergo epilepsy surgery [2]. Registration and fusion of MR and PET images will benefit the surgeon. Besides multimodality 
registration, there exist important application areas in monomodality registration. Examples include alignment of two images given in different times in order to detect silent differences. Over the years, a large number of registration techniques have been developed including point-based methods, deformation models [3] and voxel-based methods. In voxel-based approaches [4], the optimum registration is determined by iteratively optimizing a similarity measure calculated using gray values of both images. Such methods do not require user interaction but may suffer from high computational cost. When mutual information is selected as the similarity measure, estimating the joint histogram which is a time-consuming process is iteratively required. Another difficulty with this method is the possible existence of local maxima in MI function.

If original misregistration is small, the algorithm converges to the global maximum more possibly. For this reason, coarse-to-fine multi-resolution strategies have been introduced. These approaches increase accuracy of the registration and speed up the optimization algorithm. In multi-resolution methods, the pyramid of images could be obtained using the wavelet transformation [5], averaging method or image subsampling. When using image pyramids, no preference is considered for different regions of images. We propose that more samples should be selected from high entropy areas such as regions of tissue transition. Furthermore, mutual information criterion suffers from lack of spatial information. Using the proposed method, we try to compensate this shortage.

In sections 2 and 3, we first review image registration based on mutual information. Section 4 presents our proposed subsampling method and section 5 describes the search technique that we applied. In Section 6 the experimental results for registration of CT to MR and PET to MR images are presented and finally section 7 gives a summary of our method.

\section{Image Registration}

The registration problem is to find the optimal transformation $T^{*}$ which best aligns the images. For reference image $I$ and floating image $J$, image registration can be defined as follows:

$$
T^{*}=\arg \max _{T} \rho(I, T(J))
$$

where $\rho$ refers to a similarity measure. Therefore, different registration methods can be derived from different similarity measures and different search strategies. For example in MI-based methods, $\rho$ is mutual information of the images.

The most common transformations applied to register medical images are rigid and affine. An affine transformation includes transition, rotation, scaling and shearing where it maps parallel lines to parallel lines. Rigid is a special kind of affine transformation when only transitions and rotations are allowed. In rigid transformation, the objects retain their relative shape and size. It is generally used for brain images. A three-dimensional rigid transformation [6] is the product of rotation $R$ and transition $D$ matrices where the rotation matrix is the product of three matrices representing rotation around $x, y$ and $z$ axes.

$$
\begin{aligned}
T & =D \times R \\
R & =R_{x} \times R_{y} \times R_{z}
\end{aligned}
$$


Transition matrix includes three parameters $\left\{d_{x}, d_{y}, d_{z}\right\}$ as:

$$
D=\left[\begin{array}{cccc}
1 & 0 & 0 & d_{x} \\
0 & 1 & 0 & d_{y} \\
0 & 0 & 1 & d_{z} \\
0 & 0 & 0 & 1
\end{array}\right]
$$

and rotation matrices representing three angles $\left\{\varphi_{x}, \varphi_{y}, \varphi_{z}\right\}$ are expressed as:

$$
\begin{aligned}
R_{x} & =\left[\begin{array}{llrl}
1 & 0 & 0 & 0 \\
0 & \cos \left(\varphi_{x}\right) & -\sin \left(\varphi_{x}\right) & 0 \\
0 & \sin \left(\varphi_{x}\right) & \cos \left(\varphi_{x}\right) & 0 \\
0 & 0 & 0 & 1
\end{array}\right] \\
R_{y} & =\left[\begin{array}{llll}
0 & \cos \left(\varphi_{y}\right) & -\sin \left(\varphi_{y}\right) & 0 \\
0 & 1 & 0 & 0 \\
0 & \sin \left(\varphi_{y}\right) & \cos \left(\varphi_{y}\right) & 0 \\
0 & 0 & 0 & 1
\end{array}\right] \\
R_{z} & =\left[\begin{array}{llll}
0 & \cos \left(\varphi_{z}\right) & -\sin \left(\varphi_{z}\right) & 0 \\
0 & \sin \left(\varphi_{z}\right) & \cos \left(\varphi_{z}\right) & 0 \\
0 & 0 & 1 & 0 \\
0 & 0 & 0 & 1
\end{array}\right]
\end{aligned}
$$

Consequently, a rigid transformation requires six independent parameters to be determined.

\section{Mutual Information Criterion}

Mutual information is an entropy-based measure. Given $m$ events occurring with probabilities $p_{1}, \ldots, p_{m}$ the Shannon entropy is defined as:

$$
H=\sum_{i=1}^{m} p_{i} \log \frac{1}{p_{i}}=-\sum_{i=1}^{m} p_{i} \log p_{i}
$$

Entropy is a measure of uncertainty or dispersion of the probabilities of events. The Joint entropy $H(I, J)$ can be calculated using joint histogram of two images. As the images become misaligned, dispersion of their joint histogram increases. Therefore, registration of two images can be accomplished by minimizing the joint entropy of the images, but mutual information is a better criterion [7] as marginal entropies $H(I)$ and $H(J)$ are taken into account.

$$
M I(I, J)=H(I)+H(J)-H(I, J)
$$

The optimal transformation can be gained by maximizing mutual information of the two images. Normalized measure of mutual information is defined as follows:

$$
N M I(I, J)=\frac{H(I)+H(J)}{H(I, J)}
$$

Interpolation is usually required to estimate intensity values of transferred points. Nearest neighbor, linear and partial volume interpolations [8] are the most common methods for registration problem. 

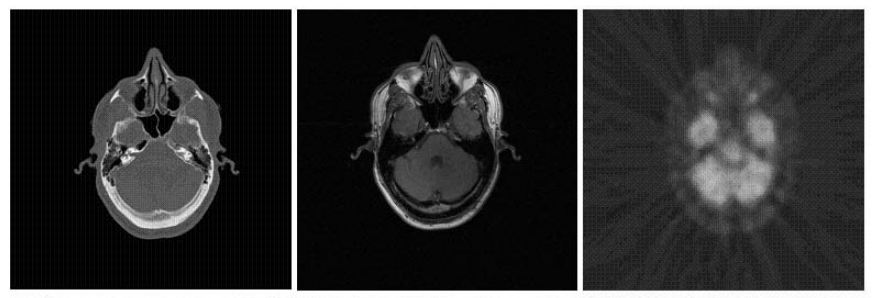

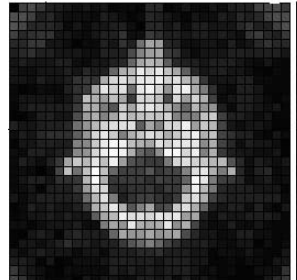

(a)

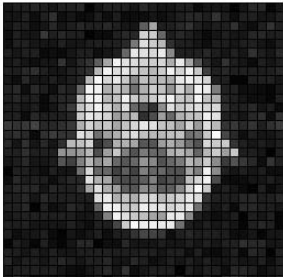

(b)

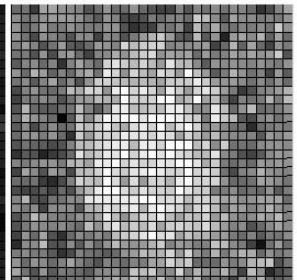

(c)

Figure 1: Entropy maps for (a) CT, (b) MR and (c) PET images. Blocks with higher entropy are shown with higher intensity.

\section{Sample Selection Method}

Optimizing MI function requires estimation of the joint histogram iteratively. Given reference image $I$ and floating image $J$ and for each transformation $T$ belonging to the search space, the joint histogram $H(I(s), J(T(s)))$ is computed for overlapping region of the images, where $s \in S$ and $S$ is the set of grid points of the reference image.

To increase speed performance, a subset of $S$ may be used, where subsampling factor $\alpha=1 / N$ means only one in $N$ voxels is selected for computing the joint histogram. The complexity of computing MI function [9] varies linearly with number of selected samples. Consequently, when subsampling factor is small, an estimation of optimal transformation can be obtained relatively fast. The result can be used as initial value in finer level (with higher $\alpha$ ).

\subsection{Variant Subsampling Factors}

The idea of the proposed method is based on using higher subsampling factors for the regions which contain more information. The common subsampling method applies a fixed factor $\alpha$ for all areas of an image. In multi-resolution techniques, subsampling is done by averaging or other methods, but again $\alpha$ is the same for all regions, whereas tissue regions deserve more attention than background. Furthermore, edge or tissue transition regions contain important information for adjustment.

A method of incorporation spatial information of edges with mutual information that was introduced in [10] uses gradient vectors of corresponding points but calculation of gradient vectors in each iteration increases the computational cost. By using variant subsampling factors, we can emphasize the role of edge regions in MI measure.

In order to measure the information content of each partition of an image, entropy is used. In Fig. 1 the entropy maps of MR, CT and PET images which divided into small blocks are shown. The block size depends on the width of edge transition regions in the 


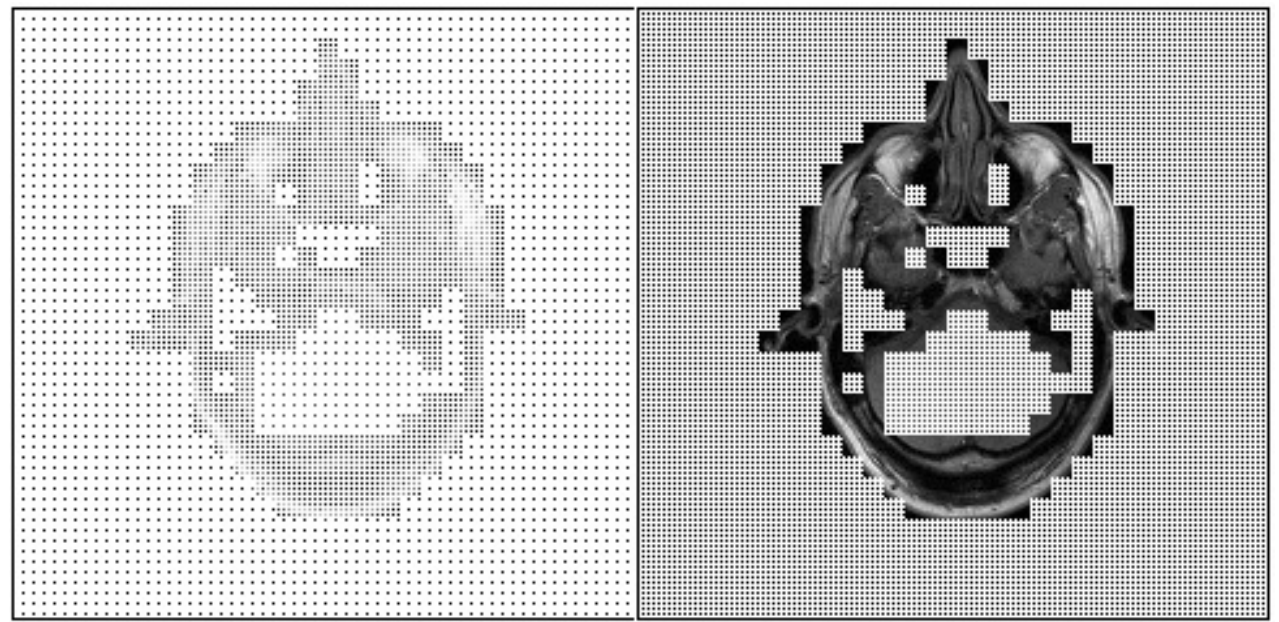

(a)

(b)

Figure 2: Selected samples using proposed method where $n=2, c=4$, (a) $\alpha_{1}=1 / 64$, $\alpha_{2}=1 / 16$ and (b) $\alpha_{1}=1 / 16, \alpha_{2}=1 / 1$.

image. For brain images of Fig. 1 and 2, blocks of $32 \times 32$ were used. Clearly, for three-dimensional images, the entropy blocks should be three-dimensional. In general, to subsample with $n$ variant factors, a series of block sets $A_{1}, \ldots, A_{n}$ in the reference image are defined as:

$$
\begin{aligned}
A_{i}=\left\{s \in B_{j} \mid \tau_{i-1}<H\left(B_{j}\right) \leq \tau_{i}\right\} \quad & i=1, \ldots, n \\
& j=1, \ldots, M \\
& \tau_{0}=0
\end{aligned}
$$

where $M$ is the number of blocks in the image, $B_{j}$ is the jth block, $H\left(B_{j}\right)$ is entropy of the jth block and $\tau_{i}$ is entropy threshold for block set $A_{i}$ which can determine how many blocks belong to the block set. For each block set $A_{i}$, a corresponding subsampling factor $\alpha_{i}$ is used which holds the condition $\tau_{p}>\tau_{q} \rightarrow \alpha_{p}>\alpha_{q}$ that means the blocks with higher entropy should be subsampled with higher factors. A simple way is to consider a constant $c$ as factor coefficient:

$$
\alpha_{i+1}=c \alpha_{i} \quad c \alpha_{i} \leq 1
$$

Fig. 2 shows selected samples for a brain MR image. Eighty percent of samples belong to the first block set $A_{1}$ with subsampling factor $\alpha_{1}$ and the rest are members of the second block set $A_{2}$ with corresponding factor $\alpha_{2}$.

A comparison of proposed method and uniform subsampling method for MR to MR registration can be found in Fig. 3. The vertical axis shows the mean of registration errors computed in several volumes of interest (VOIs) and the horizontal axis represents the percentage of samples which selected by both methods. Subjected to the voxel-size of images and the interpolation method, a minimum registration error is unavoidable. The comparison indicates that the proposed method reaches to the minimum error with a lower 
percentage of samples and yields a better registration than uniform subsampling method especially when subsampling factor is low. Fig. 4 represents MI functions for a transition and a rotation parameter, where zero position corresponds to the optimal solution. The artifacts and local maxima are reduced when using our proposed method.

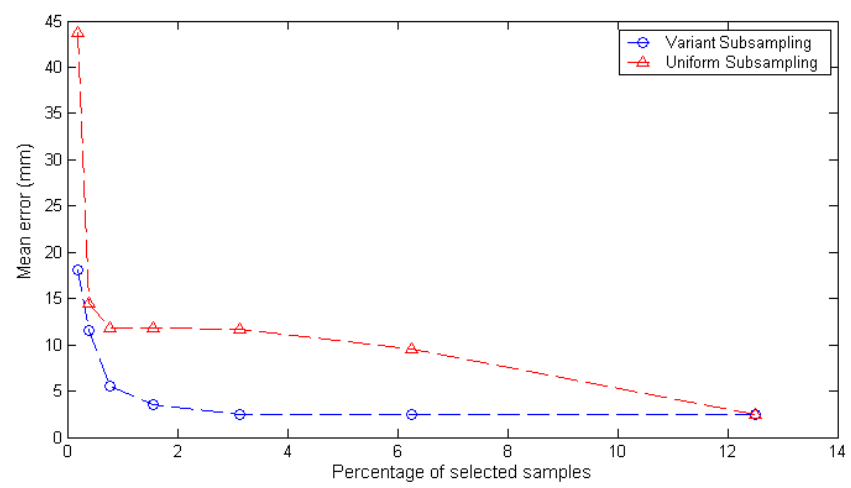

Figure 3: Circles and triangles indicate mean errors for proposed method and uniform subsampling method respectively.
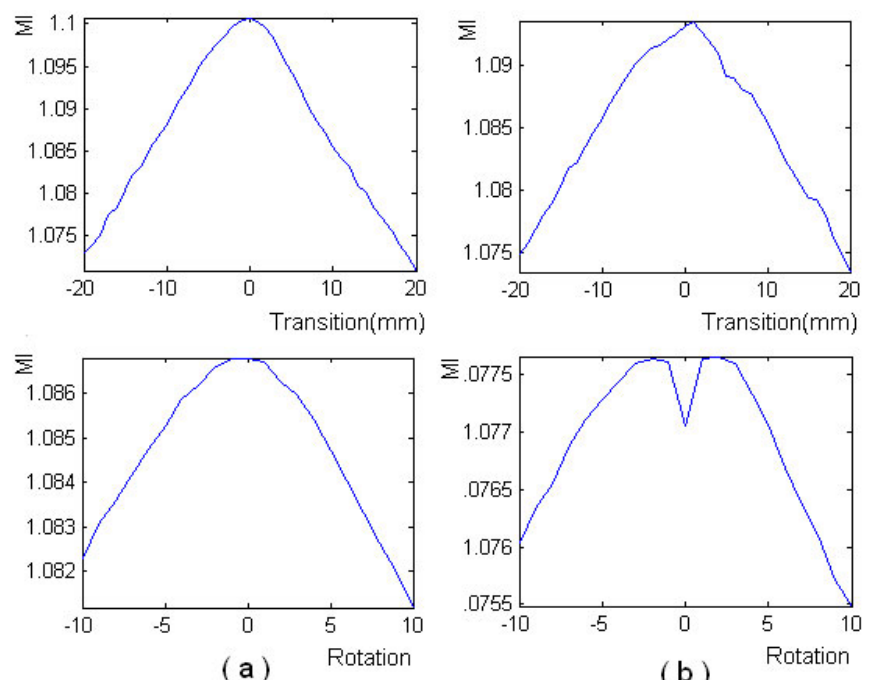

Figure 4: MI function for (a) proposed method and (b) uniform subsampling method.

\section{Search Technique}

A multi-stage strategy is used to find the optimal transformation. In each stage, MI function is computed using proposed subsampling method. The subsampling factors are small for first stage and increase gradually in higher stages. When a higher stage is started, 


\begin{tabular}{|lcccc|}
\hline Modality & $\begin{array}{c}\text { Number of } \\
\text { datasets }\end{array}$ & Median & Mean & Maximum \\
\hline \hline CT-T1 & 7 & 1.4604 & 1.5638 & 3.6254 \\
CT-T1rect & 6 & 0.6144 & 0.9798 & 5.0428 \\
CT-T2 & 7 & 2.1742 & 2.1251 & 3.3467 \\
CT-T2rect & 7 & 0.9596 & 1.2045 & 5.3249 \\
CT-PD & 7 & 2.2918 & 2.4021 & 5.0634 \\
CT-PDrect & 7 & 0.7153 & 0.9664 & 3.4570 \\
\hline
\end{tabular}

Table 1: Registration errors with respect to the gold standard for CT to MR pairs.

\begin{tabular}{|lcccc|}
\hline Modality & $\begin{array}{c}\text { Number of } \\
\text { datasets }\end{array}$ & Median & Mean & Maximum \\
\hline \hline PET-T1 & 5 & 3.1852 & 4.3604 & 9.7388 \\
PET-T1rect & 4 & 2.6150 & 2.7462 & 4.5467 \\
PET-T2 & 5 & 3.3800 & 3.4876 & 6.5244 \\
PET-T2rect & 5 & 2.9284 & 3.5646 & 7.8524 \\
PET-PD & 5 & 3.6630 & 4.4628 & 9.0148 \\
PET-PDrect & 5 & 2.9474 & 4.0269 & 9.8907 \\
\hline
\end{tabular}

Table 2: Registration errors with respect to the gold standard for PET to MR pairs.

the initial range of search space is reduced because it's expected that the algorithm has reached closer to the solution.

Simplex method and Powell's routine [11] are commonly used for registration problem. Because of existence of local maxima in MI function, these methods can not be considered as perfect optimizers. We propose using Powell's method for primary stages and a stochastic search method such as simulated annealing [12] for the final stage. Simulated annealing method is not sensitive to local maxima; however, reaching to the global maximum is not guaranteed.

\section{Experimental Results}

The proposed method was applied to register CT to MR and PET to MR volume images of Retrospective Registration Evaluation Project (RREP). MR images include T1, T2, PD and rectified versions of them. Geometrical distortions are corrected for rectified images (see [13] for more detail). Typical voxel size of the images is $(1.25 \times 1.25 \times 4)$ for MR images, $(0.65 \times 0.65 \times 4)$ for CT images and $(2.59 \times 2.59 \times 8)$ for PET images in $\mathrm{mm}$. The joint histograms were estimated using bilinear interpolation.

The difference between marker-based gold standard and our registration has been evaluated in 10 VOIs. The results are summarized in Tables 1 and 2. The mean errors are less than the largest voxel size ( $4 \mathrm{~mm}$ for CT and MR and $8 \mathrm{~mm}$ for PET), which means that all mean errors present subvoxel accuracy. Fig. 5 visualizes our results in some slices. 

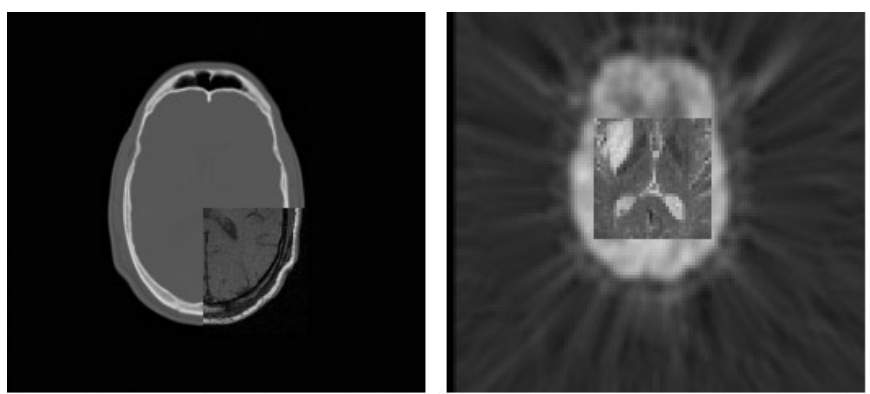

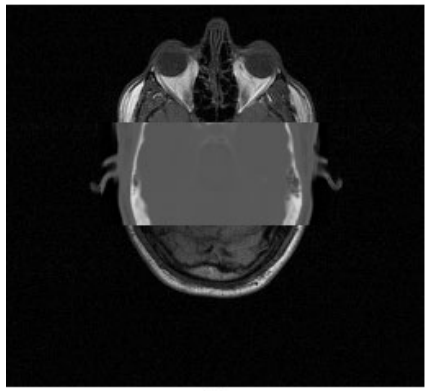

(a)

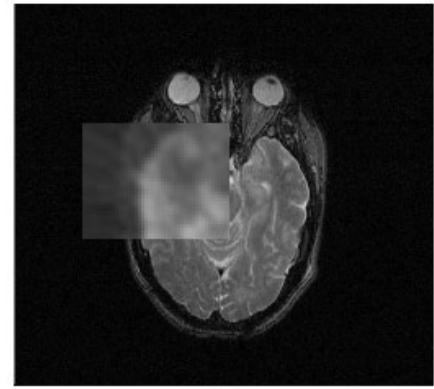

(b)

Figure 5: Fused slices of (a) CT/MR and (b) PET/MR after registration.

\section{Conclusion}

An improved multi-stage registration method based on mutual information has been introduced. The method employs a new subsampling technique which selects more relevant samples for joint histogram estimation. Because of existence of local maxima, simulated annealing is used in the final stage to increase the chance of finding the optimal solution.

The method is tested for volume image pairs of CT/MR and PET/MR. The registration errors are evaluated with respect to the marker-based gold standard. The results represent subvoxel accuracy for all mean errors.

\section{Acknowledgements}

The images and gold standard transformations were provided as part of the project, "Retrospective Image Registration Evaluation", National Institutes of Health, Project Number 8R01EB002124-03, Principal Investigator, J. Michael Fitzpatrick, Vanderbilt University, Nashville, TN, USA.

This work was supported by Iran Telecommunication Research Center (ITRC), under grant T-500-1826.

\section{References}

[1] Lisa G. Brown. A survey of image registration techniques. ACM Computing Surveys, 24(4):325-376, 1992. 
[2] B. A. Maintz and M. A. Viergever. A survey of medical image registration. Medical Image Analysis, 2(1):1-36, Apr 1998.

[3] V. Noblet, C. Heinrich, F. Heitz, and J. Armspach. 3-d deformable image registration: a topology preservation scheme based on hierarchical deformation models and interval analysis optimization. IEEE Transactions on Image Processing, 14(5):553$566,2005$.

[4] T. Netsch, P. Rosch, A. Muiswinkel, and J. Weese. Towards real-time multi-modality $3 \mathrm{~d}$ medical image registration. In Proceedings of the Eighth International Conference On Computer Vision, pages 718-725, 2001.

[5] Hua-mei Chen and Pramod K. Varshney. A pyramid approach for multimodality image registration based on mutual information. Proceedings of 3 rd international conference on information fusion, I:9-15, July 2000.

[6] R. Shekhar and V. Zagrodsky. Mutual information-based rigid and nonrigid registration of ultrasound volumes. IEEE Transactions on Medical Imaging, 21(1):9-22, 2002.

[7] Josien P. W. Pluim, J. B. Antoine Maintz, and Max A. Viergever. Mutual information based registration of medical images: a survey. IEEE Transactions on Medical Imaging, 22(8):986-1004, 2003.

[8] Jeffrey Tsao. Interpolation artifacts in multimodality image registration based on maximization of mutual information. IEEE Transactions on Medical Imaging, 22(7):854-863, July 2003.

[9] F. Maes and A. Collignon. Multimodality image registration by maximization of mutual information. IEEE Transactions on Medical Imaging, 16(2):187-198, Apr 1997.

[10] J. P. W. Pluim, J. B. A. Maintz, and M. A. Viergever. Image registration by maximization of combined mutual information and gradient information. IEEE Transactions on Medical Imaging, 19(8):809-814, 2000.

[11] W. H. Press, B. P. Flannery, S. A. Teukolsky, and W. T. Vetterling. Numerical recipes in C. Cambridge University Press, Cambridge, UK, 1992.

[12] N. Ritter, R. Owens, J. Cooper, R. H. Eikelboom, and P. P. Van Saarloos. Registration of stereo and temporal images of the retina. IEEE Transactions on Medical Imaging, 18(5):404-418, May 1999.

[13] J. West and J. M. Fitzpatrick. Comparison and evaluation of retrospective intermodality brain image registration techniques. Journal of Computer Assisted Tomography, 21(4):554-566, 1997. 